\title{
Application and development of CT scanning technology in coal sector
}

\author{
Xiangjie Qin, ${ }^{\mathrm{a}, \mathrm{b}}$, Gang Wang ${ }^{\mathrm{a}, \mathrm{b},{ }^{*}, \text { Dongyang Han }}{ }^{\mathrm{a}, \mathrm{b}}$, and Xuechang Chen ${ }^{\mathrm{a}, \mathrm{b}}$ \\ ${ }^{a}$ Shandong University of Science and Technology, Mine Disaster Prevention and Control-Ministry of \\ State Key Laboratory Breeding Base, Qingdao 266590, PR China \\ ${ }^{\mathrm{b}}$ Shandong University of Science and Technology, College of Mining and Safety Engineering, \\ Qingdao 266590, PR China
}

\begin{abstract}
CT scanning technology has been widely used in the observation of coal microstructures because of its advantages of nondestructive and refined visualization. In this study, the post-processing methods (image processing, 3D reconstruction, numerical simulation, etc.) of CT scan images are introduced in detail, and the application of CT in the coal sector is systematically reviewed, mainly including: coal seam gas mining (coal structure, reservoir permeability etc.); mechanical properties of coal mass damage; coal seam water injection, coal mass permeability enhancement, coal washability, etc. The application of CT in various research fields has been relatively mature. The initial research is focused on the analysis of coal mass structure, and subsequently the fractal dimension is introduced and the in-depth study of coal mass seepage is involved. At the same time, CT scanning equipment is been constantly improving to be applied to a wider range of explorations. In the future, improved image processing or equipment can increase the research accuracy, and the field of coal structure and seepage under the multi-field coupling conditions such as pressure-temperature-seepage can be explored.
\end{abstract}

\section{Introduction}

As a porous medium, coal has interconnected and intricate internal pore and fissure structures, which makes it difficult to reveal the storage and migration mechanism of coal pore and fissure structures $[1,2]$. The current research methods for the microstructure of coal mass can be summarized into two categories. One is the method of physical experiments, mainly including the mercury injection method and the low temperature nitrogen adsorption method, etc. [3, 4] Those two observation methods are similar in principle, and they are both used to analyze the characteristics of coal porosity and connectivity based on the mercury entry / nitrogen adsorption and mercury desorption / nitrogen desorption curves. The other category is the direct observation method, which mainly includes Computed Tomography (CT) [5], Scanning Electron Microscope (SEM) [6], Focused Ion Beam (FIB) [7], Nuclear Magnetic Resonance (NMR) [8], etc. The most

*Corresponding author: ipo@sdu.edu.cn 
significant advantage of those methods is visual observation of the microstructure of coal mass under non-destructive conditions. Among those methods, FIB and SEM are similar and they can only be used to observe the surface of the object under observation while the observation methods of CT and NMR can achieve 3D observation of the microstructure of coal mass. In comparison, the observation scale of NMR is better than that of CT. However, $\mathrm{CT}$ can realize the quantitative description of coal microstructures and seepage characteristics through post-processing and 3D reconstruction technology with the help of $3 \mathrm{D}$ reconstruction software and simulation software. Furthermore, it has stronger data postprocessing capabilities. In this paper, a set of technical processes from CT scanning, 3D reconstruction to numerical simulation is introduced in detail, the application and development of this technical method in various fields of coal microstructure research is summarized, and the future research direction is proposed and suggested.

\section{Post processing technology based on CT images}

\section{D reconstruction technology}

The process of 3D CT reconstruction can be divided into four steps: image selection, filtering algorithm, image segmentation, and 3D reconstruction. (1) Image selection refers to the selection of the area for study and analysis. Since the volume data acquired by a CT scan occupies a large amount of memory, it is not conducive to later analysis. Based on the self-similar characteristics of coal mass, a suitable size is selected for study at which the reconstructed model has similar properties to coal samples, and thus it is also called Representative Elementary Volume (REV). At present, it is generally believed that when the size of the research object is $200 \times 200 \times 200$ pixel $^{3}$, the structural parameters of the model remain basically unchanged with the increase of the model volume $[9,10]$. (2) Because a large amount of system noise in the image will affect the accuracy of the reconstruction results, filtering is required. The commonly used filtering algorithms are Gaussian filtering, median filtering, and mean filtering. Since median filtering is better for processing salt and pepper noises, median filtering is currently used for noise reduction in most studies. (3) Image segmentation is the most important step in the 3D reconstruction process. The image segmentation technology is used to extract different structures (pores and fissures, coal matrix, high-density mineral components, etc.) in images, and thus the quality of the image segmentation will directly affect the accuracy of reconstruction results. Image segmentation methods can be divided into two categories. One is the threshold segmentation method, i.e., setting a gray value range according to the gray values of an image to extract the target object. The other is the area-based segmentation method, such as watershed algorithm. (4) 3D visualization of different structures can be realized by applying the volume rendering module in the $3 \mathrm{D}$ reconstruction software to a series of $2 \mathrm{D} \mathrm{CT}$ images after filtering and image segmentation. Fig. 1 shows the 3D CT reconstruction process. 


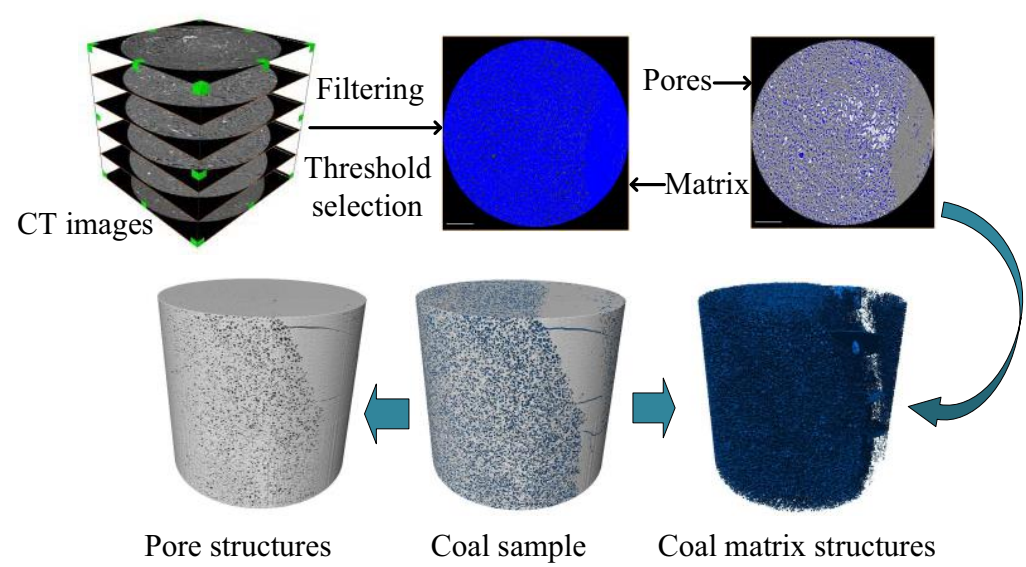

Fig. 1. 3D CT reconstruction process based on AVIZO software

Fractal dimension of 3D pore and fissure structure model

Due to the fractal characteristics of the pore and fissure structure of coal mass, fractal dimension can be used to describe the complexity of the pore and fissure structure of coal mass quantitatively. At present, the 2D and 3D fractal dimension of a CT image is mostly calculated by using the box counting algorithm, whose principle is to divide the fractal body into uniform grids (the grid side length is $\varepsilon$ ), calculate the number of grids $N$ required to cover the entire fractal body, and gradually refine the grids with the number of required grids recorded so as to calculate the fractal dimension.

$$
\operatorname{dim}_{\text {box }}(S)=\lim _{s \rightarrow 0} \frac{\log N(s)}{\log (1 / s)}
$$

In the calculation process, the linear relationship between $\log (N)$ and $\log (1 / \varepsilon)$ is usually drawn through software (matlab, AVIZO, etc.), and the slope of the line is the fractal dimension.

$$
\log (N(s))=\log (C)+F D \log (1 / s)
$$

Numerical simulation based on 3D reconstruction model

Here the realization process of seepage simulation by the $3 \mathrm{D}$ reconstruction software AVIZO and the seepage simulation software ANSYS-CFX is introduced as an example. First, based on the model construction method in Section 2.1, the pore and fissure structure of coal mass is extracted, the model for the pore and fissure structure of coal mass is established, and the interconnected pore and fissure structure model is extracted with the isolated pores removed. Then, the interconnected pore and fissure structure model is meshed. Since AVIZO has the function of grid generation, the grid generation and grid attribute setting can be achieved in the software. In addition, the structural model can be exported from AVIZO and meshed using other software such as ICEM. Next, the meshed model is imported into ANSYS-CFX, and the setting of boundary conditions is completed 
in CFX, mainly including Inlet, Outlet, Wall, etc. The fluid attribute can be set to water or methane, and the inlet and outlet boundary conditions can be set to inlet pressure, outlet pressure or inlet speed, outlet pressure. The setting of specific values is based on the actual situation. Finally, the solving parameters such as the number of iteration steps and error precision are set, and the solving process is run. After solving, the CFX-Post in ANSYS can be used to extract the simulation results (including fluid velocity, pressure, mass flow and other parameters).

In addition to the above seepage simulation methods, the Lattice Boltzmann (LBM) method can also be used for simulation [11]. Moreover, a series of research can be realized by the combination of coal skeleton models and simulation software. For example, the Hypermesh software is used for dynamic seepage simulations in coal skeletons based on CT reconstructed coal skeleton models, and the discrete element analysis software FLAC ${ }^{3 \mathrm{D}}$ is used to apply pressure to coal skeleton models to explore the evolution and mechanical characteristics of coal pore and fissure structure under different pressure conditions.

\section{Application of CT scanning technology in coal sector}

\section{Coalbed methane mining (mechanism of gas storage and migration)}

In order to reveal the mechanism of gas storage and migration in the microstructures of coal, based on CT 3D reconstruction technology, the microstructure of coal can be characterized qualitatively and quantitatively. The structural characteristics such as pore size distribution and connectivity are analyzed by development of equivalent pore and fissure network models [12]. For improving the scanning accuracy, synchrotron radiation nano-CT can be adopted to scan coal particles and reconstruct them three-dimensionally for analysis of the pore and fissure structure of coal mass in nm scale [13]. Since gas contrast agents can easily penetrate narrow pore and fissure networks without surface tension problems, xenon and krypton are used to study gas adsorption in coal, and K-edge subtraction is adopted to achieve selective imaging of specific chemical elements to obtain more accurate porosity [14], Fig.2 shows the imaging structure of Xenon after diffusion.
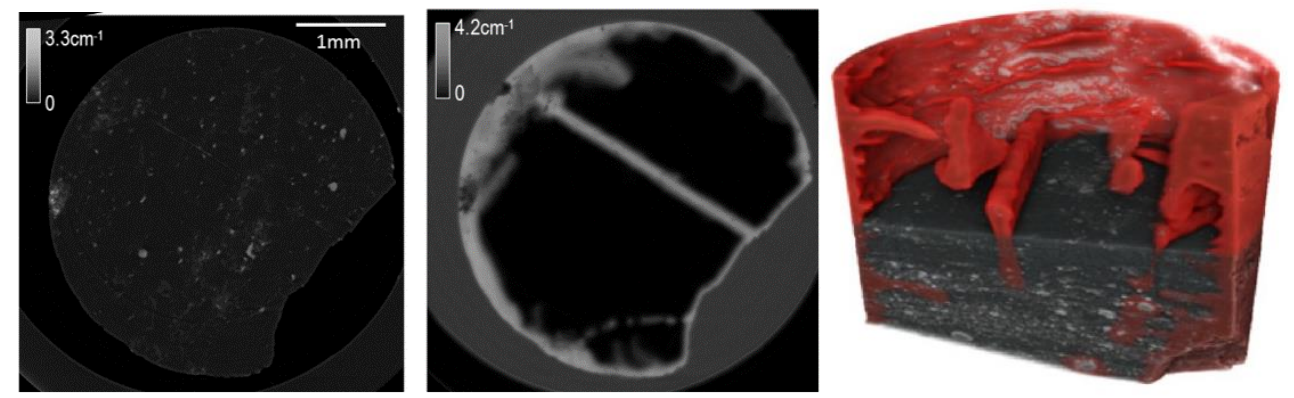

Fig. 2. From left to right: Original image, xenon diffused image, and reconstructed image (xenon distribution is shown in red)

In subsequent research, the $\mathrm{CT}$ reconstructed model is combined with finite element seepage simulation software for the study of gas seepage characteristics in the microstructure of coal. The meshed model is imported into the simulation software, and the seepage simulation is performed in the three directions of X, Y, Z [15]. Then the absolute permeability in the three directions is calculated based on Darcy's law, and thus the anisotropy of coal mass is proved. It is also possible to construct pores of different shapes, different sizes and different positions on CT images artificially by applying the principle of 
different gray values on CT images corresponding to different structures of coal, and study the influence of pore size, position and shape on gas seepage [16]. In order to solve the problem of low resolution of CT images, the subvoxel processing algorithm based on matlab is used to improve the resolution of coal pore and fissure structures, and the LBM algorithm is applied for the simulation of the permeability of coal [17]. Fractal dimension can be incorporated into the exploration of coal structures and seepage characteristics according to the fractal characteristics of the pore and fissure structure of coal mass, and the qualitative and quantitative relationship between fractal dimension and porosity or permeability is established [18]. Moreover, CT is applied for scanning coal of different ranks and the fractal dimension is calculated with the box counting algorithm combined to study the structural characteristics of coal mass with different vitrinite contents. It is found that the average micro fissure length, width and volume decrease while the number increases as the coal rank increases [19]. Furthermore, the porosity shows a U-shaped trend [20]. Larger fractal dimensions of coal mass correspond to poorer gas permeability, which is not conducive to CBM exploitation.

Research of mechanical properties of coal damage

With the maturity and development of 3D CT reconstruction technology, its application in the study of coal mechanics and damage characteristics is becoming more and more extensive. The initial research process is as follows: first conduct compression experiments on coal mass; then observe the damage of coal mass by using CT equipment; finally observe the damage and fractal characteristics of coal mass under different confining pressures through 3D reconstruction [21]. Later CT equipment is improved and loading under uniaxial compression or triaxial confining pressure is achieved. The coal samples in the non-unloading state are scanned, and then loading is continued and the coal samples are scanned again [22]. For example, Zhong JC. et al. [23] studied structural damage evolution characteristics of coal under uniaxial compression by their self-developed uniaxial compression equipment in combination with CT scanning equipment. Zhou HW. et al. [24] scanned the coal samples through the CT scanning equipment with uniaxial compression and analyzed the porosity and permeability characteristics of the coal samples under uniaxial compression with the simplified fractal model for the fractured coal combined. Li YY. et al. [25] used the CT scanning system with triaxial compression equipment for their CT scanning experiments on the anthracite under uniaxial and triaxial compression conditions, and describe the destructive evolution process quantitatively through parameters such as the gray value, fissure volume and CT porosity.

In addition, numerical simulation can also be performed based on 3D CT reconstructed model. Wang G. et al. [10] conducted uniaxial compression experiments on the coal skeleton by using hypermesh and LS-DYNA, and performed seepage simulations based on the compressed model to investigate the seepage characteristics of coal mass under different loading speeds. Zhong JC. et al. [23] developed a numerical computational model for distinguishing coal matrix and coal impurities in the FLAC ${ }^{3 \mathrm{D}}$ software for the numerical simulation under uniaxial and triaxial loading conditions.

\section{Coal seam water injection, permeability enhancement, and}

\section{coal washability, etc.}

Coal seam water injection is an effective measure to prevent bursts and dust. Water injection seepage in coal microstructures can be simulated based on the reconstructed model of coal mass, and the coal skeleton structure can be reconstructed threedimensionally by the reconstruction software based on matlab. The mesh generation and boundary condition setting can be performed in Hypermesh, and the ALE algorithm can be 
used for solution. In this way the dynamic seepage simulation can be realized based on the coal skeleton. Furthermore, in order to realize the water injection seepage simulation in high temperature environment, AVIZO can be used to construct the coal pore and fissure structure model and the coal skeleton structure model, respectively. The thermal boundary conditions can be set in ANSYS-CFX to study the fluid-solid coupling heat transfer in coal mass $[26,27]$.

Low permeability of coal is the key reason for restricting coal seam water injection and coalbed methane exploitation. However, the permeability of coal after high and low temperature impact can be greatly improved. The cold and hot impact tests are carried out on coal mass through high and low temperature experimental systems before CT scanning experiments. Then the coal pore and fissure structure model is established through the VGStudio MAX image analysis software [28], as shown in Fig.3. The evolution characteristics of the pore and fissure structure of coal mass is analyzed quantitatively. On this basis, different confining pressures are applied to coal mass after temperature impact. The seepage of coal under different confining pressures after temperature impact can be analyzed with the gas permeability experimental device combined [29]. Supercritical $\mathrm{CO}_{2}$ fracturing has a similar effect to hydraulic fracturing. The specimens are scanned by CT before and after coal impact [30], and the structure before and after impact is reconstructed to build an equivalent pore and fissure network model for the study of the change in structural parameters such as porosity, interconnected pore volume, and number of pore throats after fracturing [31]. Due to the deep mining of coal, there is an increasing need for safe and efficient coal mining methods and water jet coal breaking technology is more widely studied. CT scanning technology can be used to reconstruct the structure of coal mass after the impact of the water jet. It is found that the kinetic energy of the water jet is gradually consumed in the vertical direction and that both the damage range and the damage degree decrease with the increase of the standard depth [32].

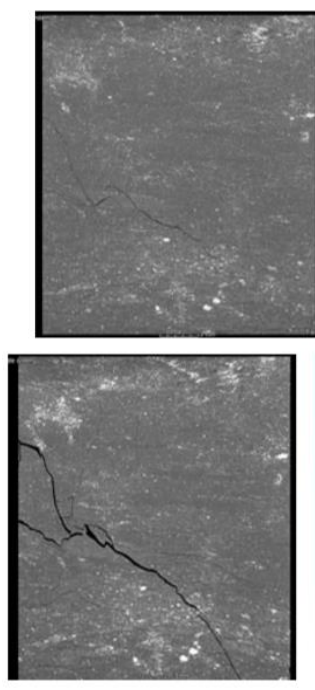

(a) Before temperature shock

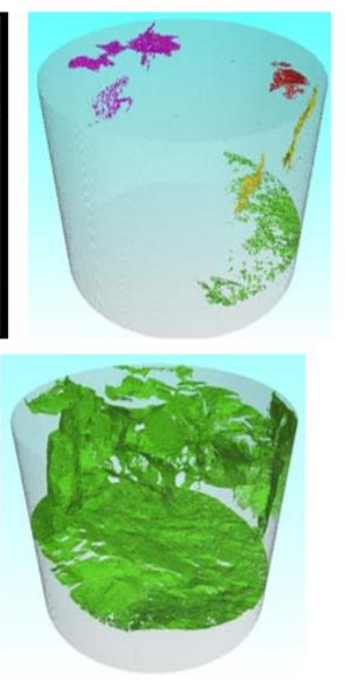

(b) After temperature shock

Fig. 3. Change in coal structure under high and low temperature impact

In addition, some research is focused on the prediction of coal washability by CT scanning technology [33]. The coal after drop tests is scanned repeatedly by CT, and the 3D 
watershed algorithm and segmentation algorithm are used to monitor the size and distribution of coal fragments and calculate the density of each fragment in order to obtain coal washability. The results are consistent with the test results of Float and Sink (FaS).

\section{Research prospect}

Currently, CT scanning technology has been widely applied and popularized in the study of the micro pore and fissure structures and seepage characteristics of coal. In current research, 3D reconstruction software and numerical simulation methods are mainly adopted to apply CT to various coal research fields. The processing and utilization of CT reconstructed models are relatively mature. Furthermore, the CT scanning system with uniaxial compression and triaxial compression has emerged to realize CT scanning in complex environments.

In future research, the focus can be the improvement of CT accuracy and the improvement of CT equipment. Because the scale of CT observation is currently concentrated on the $\mu \mathrm{m}$ scale and it is limited by the sample size, the improvement of CT accuracy through image processing is an important research direction. The improvement of CT equipment is another important direction. The improved CT equipment for real-time scanning and dynamic observation of the evolution of coal structures in complex environments needs to be developed. For example, it can be used for dynamic observation under temperature conditions or even temperature-pressure-seepage coupling conditions, which are more in line with the coal storage environment with a high burial depth. While experiments are conducted, numerical simulations can be performed with CT reconstructed models to verify the results from experiments. However, as a research method of observation, CT scanning technology also has its own defects and it is not applicable to all research. At present, many researchers combine CT with other observation methods (ATR-FTIR, FIB-SEM, etc.) [3537] to achieve a more comprehensive study. Therefore, some other observation methods may be used reasonably, not just limited to CT observation methods.

\section{Acknowledgments}

This work was supported by the National Natural Science Foundation of China (Project No. 51934004; 51674158; 51574158; 51474106), the Major Program of Shandong Province Natural Science Foundation (ZR2018ZA0602), the Taishan Scholar Talent Team Support Plan for Advantaged \& Unique Discipline Areas, the Science and technology support plan for Youth Innovation of colleges and universities in Shandong Province (2019KJH006).

\section{References}

1. Yi. Tarasevich, Porous structure and adsorption properties of natural porous coal. Colloids Surfaces A Physicochem Eng Asp, 176:267, 72 (2001)

2. Z. Qin, New advances in coal structure model. Int J Min Sci Technol, 28:541, 59 (2018)

3. Cai Y, Li Q, Liu D, Zhou Y, Lv D. Insights into matrix compressibility of coals by mercury intrusion porosimetry and N2 adsorption. Int J Coal Geol, 200, 199-212 (2018)

4. Qin L., Li S., Zhai C., Lin H., Zhao P., Yan M., et al. Joint analysis of pores in low, intermediate, and high rank coals using mercury intrusion, nitrogen adsorption, and nuclear magnetic resonance. Powder Technol 362:615, 27 (2020) 
5. Saxena N., Hows A., Hofmann R., Alpak FO., Dietderich J., Appel M., et al. Rock properties from micro-CT images: Digital rock transforms for resolution, pore volume, and field of view, Adv Water Resour, 134, 103419 (2019)

6. Xu H., Wang G., Fan C., Liu X., Wu M.. Grain-scale reconstruction and simulation of coal mechanical deformation and failure behaviors using combined SEM Digital Rock data and DEM simulator, Powder Technol, 360:1305, 20 (2020)

7. Garnier A., Filoni G., Hrnčír T., Hladík L., Plasma FIB: Enlarge your field of view and your field of applications, Microelectron Reliab, 55:2135, 41 (2015)

8. Zhao Y., Sun Y., Liu S., Wang K.., Jiang Y., Pore structure characterization of coal by NMR cryoporometry, 190:359, 69 (2017)

9. Ni X., Miao J., Lv R., Lin X., Quantitative 3D spatial characterization and flow simulation of coal macropores based on MCT technology, 200:199, 207 (2017)

10. Wang G., Jiang C., Shen J., Han D., Qin X.. Deformation and water transport behaviors study of heterogenous coal using CT-based 3D simulation. Int J Coal Geol., 211, 103204 (2019)

11. Zhao Y., Wang Z., Ye J., Sun H., Gu J., Lattice Boltzmann simulation of gas flow and permeability prediction in coal fracture networks, J Nat Gas Sci Eng, 53:153, 62 (2018)

12. G.Wang, J.Shen, X.Chu, C.Cao, C.Jiang. Characterization and analysis of pores and fissures of high-rank coal based on CT three-dimensional reconstruction. J CHINA COAL, 42:2074, 80 (2017)

13. Zhao Y., Sun Y., Liu S., Chen Z., Yuan L., Pore structure characterization of coal by synchrotron radiation nano-CT. Fuel, 215:102, 10 (2018)

14. Mayo S., Josh M., Nesterets Y., Esteban L., Pervukhina M., Clennell M Ben, et al., Quantitative micro-porosity characterization using synchrotron micro-CT and xenon $K$ edge subtraction in sandstones, carbonates, shales and coal, Fuel, 154:167, 73 (2015)

15. Wang G., Yang X., Zhang X., Xue J., Li W., Establishment of digital coal model using computed tomography based on reverse engineering technology and three-dimensional reconstruction, Rock Soil Mech 36:3322, 44 (2015)

16. Wang G., Chu X., Yang X. Numerical simulation of gas flow in artificial fracture coal by three-dimensional reconstruction based on computed tomography. J Nat Gas Sci Eng, 34:823, 31 (2016)

17. Roslin A., Pokrajac D., Zhou Y. Cleat structure analysis and permeability simulation of coal samples based on micro-computed tomography (micro-CT) and scan electron microscopy (SEM) technology, 254, 115579 (2019)

18. Wang G., Shen J., Liu S., Jiang C., Qin X., Three-dimensional modeling and analysis of macro-pore structure of coal using combined X-ray CT imaging and fractal theory, Int J Rock Mech Min Sci, 123, 104082 (2019)

19. Heriawan M.N., Koike K., Coal quality related to microfractures identified by CT image analysis. Int J Coal Geol, 140:97, 110 (2015)

20. Shi X., Pan J., Hou Q., Jin Y., Wang Z., Niu Q., et al., Micrometer-scale fractures in coal related to coal rank based on micro-CT scanning and fractal theory, 212:162, 72 (2018)

21. Li G., Zhang R, Xu X.L., Zhang Y.F.. CT image reconstruction of coal rock threedimensional fractures and body fractal dimension under triaxial compression test, Rock Soil Mech, 36:1633, 42 (2015)

22. Nie B., He X., Li X., Chen W., Hu S., Meso-structures evolution rules of coal fracture with the computerized tomography scanning method. Eng Fail Anal, 41:81, 8 (2014)

23. J. Zhong, Z. Wang, L.Wang, J. Zhao, W. Ren, H. Zhou. Characteristics of damage evolution of deep coal based on CT three-dimensional reconstruction. J CHINA COAL Soc, 44:1482, 94 (2019) 
24. Zhou H.W., Zhong J.C., Ren W.G., Wang X.Y., Yi H.Y., Characterization of porefracture networks and their evolution at various measurement scales in coal samples using X-ray $\mu \mathrm{CT}$ and a fractal method. Int J Coal Geol, 189:35, 49 (2018)

25. Li Y., Cui H., Zhang P., Wang D., Wei J.. Three-dimensional visualization and quantitative characterization of coal fracture dynamic evolution under uniaxial and triaxial compression based on $\mu \mathrm{CT}$ scanning, Fuel, 262, 116568 (2020)

26. Wang G., Qin X.J., Jiang C.H., Zhang Z.Y., Seepage and deformation simulation of CT $3 \mathrm{D}$ reconstruction of coal microstructure under temperature. Rock Soil Mech, 41:1, 11 (2020)

27. Wang G., Jiang C., Liu S., Chu X., Shen J., Dynamic simulation of seepage process based on CT 3D reconstruction of coal skeleton structure model. J China Coal Soc, 43:1390, 1399 (2018)

28. Wang D., Zhang P., Pu H., Wei J., Liu S.. Experimental research on cracking process of coal under temperature variation with industrial micro-CT. Chinese J Rock Mech Eng, 37:10, 2243 (2018)

29. Wang D., Zhang P., Wei J., Yu C., The seepage properties and permeability enhancement mechanism in coal under temperature shocks during unloading confining pressures, J Nat Gas Sci Eng. 77, 103242 (2020)

30. Lu Y., Liao Y., Tang J., Zhang X., Han S., Ling Y., Experimental study on fracture initiation pressure and morphology in shale using supercritical $\mathrm{CO} 2$ fracturing, Journal China Coal Soc, 43:175, 80 (2017)

31. Du Y., Sang S., Pan Z., Wang W., Liu S., Fu C., Experimental study of supercritical $\mathrm{CO} 2-\mathrm{H} 2 \mathrm{O}-$ coal interactions and the effect on coal permeability, Fuel, 253:369, 82 (2019)

32. Xue Y., Si H., Yang Z., Xu D., Microscopic damage field in coal induced by water jets. J Loss Prev Process Ind. 56:300, 15 (2018)

33. Nguyen T.D, Nguyen A.V., Lin C-L., Miller J.D., Application of high-resolution X-ray microcomputed tomography for coal washability analysis. Miner Eng, 124:137, 48 (2017)

34. Liu H., Rodrigues S., Shi F., Esterle J., Manlapig E., Coal washability analysis using Xray tomographic images for different lithotypes, Fuel, 209:162, 71 (2017)

35. Li Z, Liu D, Cai Y, Ranjith PG, Yao Y. Multi-scale quantitative characterization of 3-D pore-fracture networks in bituminous and anthracite coals using FIB-SEM tomography and X-ray M-CT. Fuel, 209:43, 53 (2017)

36. Lee S., Yu J., Mahoney M., Tremain P., Moghtaderi B., Tahmasebi A., A study on the structural transition in the plastic layer during coking of Australian coking coals using Synchrotron micro-CT and ATR-FTIR., Fuel, 233:877, 84 (2018)

37. Wu H., Yao Y., Zhou Y., Qiu F., Analyses of representative elementary volume for coal using X-ray M-CT and FIB-SEM and its application in permeability predication model, Fuel, 254, 115563 (2019) 